\section{Comments of the Mismatched/Missing References in Published Article}

\author{
Ekim Onur Orhan'1, Özgür Irmak²
}

\section{Letter to Editor}

\section{Dear Editor,}

First of all, we would like to congratulate the authors of the review article by Gomes et al (1) entitled "Chlorhexidine in Endodontics", which was published in Brazilian Dental Journal in 2013. Secondly, we would like to comment on an specific aspect of this review. We have noticed mismatched/ missing references in the article. Due to the impact of this review in the literature, and also due to the importance of the mismatched/missing references, it is interesting to mention it to authors and readership.

In our understanding, reference \#4, which was mentioned in pages 93 and 94, should actually be two different references, as follows:

In page 93, reference \#4 (named 4a below) (2), should be: Prado M, Santos Junior HM, Rezende CM, Pinto AC, Faria RB, Simão RA, Gomes BP. "Interactions between irrigants commonly used in endodontic practice: a chemical analysis. J Endod 2013;39:505-510.

"Prado et al (4a) investigated the byproducts formed in the associations between the most commonly used irrigants in the endodontic practice, through electrospray ionization quadrupole time-of-flight mass spectrometry (ESI-OTOF-MS) analyses. Regarding the CHX associations, $2 \% \mathrm{CHX}$ gel and solution immediately produced an orangebrown precipitate when associated with 1\%, 2.5\% and $5.25 \% \mathrm{NaOCl}$ solutions, and an orange-white precipitate, when associated with $0.16 \% \mathrm{NaOCl}$. When associated with EDTA, CHX produced a white-milky precipitate, related to the acid-base reactions. When associated with saline and ethanol, a salt precipitation was produced. No precipitate was observed when CHX was associated with distilled water, citric acid or phosphoric acid.

Regarding the orange-brown precipitate, it occurs due the presence of $\mathrm{NaOCl}$, an oxidizing agent causing chlorination of the guanidino nitrogens of the $\mathrm{CHX}$ (4a). Basrani et al. (82) detected the presence of parachloroaniline (PCA) in this precipitate. On the other hand, Thomas \& Sem (83), Nowicki \& Sem (84) and Prado et al. (4a) failed to detect it using different methodologies. PCA has been found to be mutagenic in microorganisms

\author{
'Department of Endodontics, Faculty \\ of Dentistry, Eskisehir Osmangazi \\ University, Eskisehir, Turkey \\ ${ }^{2}$ Department of Restorative Dentistry, \\ Faculty of Dentistry, Eskisehir \\ Osmangazi University Eskisehir, Turkey \\ Correspondence: Özgür \\ Irmak, Eskisehir Osmangazi \\ University, Eskisehir, 26480, \\ Turkey. Tel: XXXXXXXXX \\ e-mail: zgrrmk@gmail.com
}

$(14,85)$ and cytotoxic $(80)$. Some concern over possible carcinogenicity also has been expressed (82)."

However, in page 94 reference \#4 (named $4 \mathrm{~b}$ below) (3) should be: Prado M, Simão RA, Gomes BP. Effect of different irrigation protocols on resin sealer bond strength to dentin. J Endod 2013;39:689-692

"De Assis et al. (107) observed that a final flush with CHX favor the wettability of AH Plus and Real Seal SE sealers on dentine surface. Additionally, Hashem et al. (108) verified that the bond strength of ActiV GP was improved by using $2 \%$ CHX in the final irrigation after $17 \%$ EDTA. Prado et al. (4b) found that the irrigation protocols influenced the bond strength of the resin sealers to dentine. In the gutta-percha/ AH Plus groups, the bond strength was higher when $\mathrm{NaOCl}$ was associated with phosphoric acid or the CHX with EDTA; In Resilon/Real Seal SE groups, the protocol associating $\mathrm{CHX}$ with phosphoric acid showed better results. The use of $\mathrm{CHX}$ as final irrigant did not negatively affect the bond strength."

We hope that you would find our comments relevant enough to find their place in your journal.

\section{References}

1. Gomes BP, Vianna ME, Zaia AA, Almeida JF, Souza-Filho FJ, Ferraz CC. Chlorhexidine in Endodontics. Braz Dent J 2013; 24:89-102.

2 Prado $M$, Santos Junior $H M$, Rezende $C M$, Pinto $A C$, Faria $R B$, Simão RA, Gomes BP. "Interactions between irrigants commonly used in endodontic practice: a chemical analysis. J Endod 2013;39:505-510.

3. Prado $M$, Simão RA, Gomes BP. Effect of different irrigation protocols on resin sealer bond strength to dentin. J Endod 2013;39:689-692. 